\title{
The arithmetic derivative and Leibniz-additive functions
}

\section{Pentti Haukkanen $^{1}$, Jorma K. Merikoski ${ }^{1}$ \\ and Timo Tossavainen ${ }^{2}$}

${ }^{1}$ Faculty of Natural Sciences

FI-33014 University of Tampere, Finland

e-mails: pentti.haukkanen@uta.fi, jorma.merikoski@uta.fi

${ }^{2}$ Department of Arts, Communication and Education

Lulea University of Technology, SE-97187 Lulea, Sweden

e-mail: timo.tossavainen@ltu.se

Abstract: An arithmetic function $f$ is Leibniz-additive if there is a completely multiplicative function $h_{f}$ such that

$$
f(m n)=f(m) h_{f}(n)+f(n) h_{f}(m)
$$

for all positive integers $m$ and $n$. A motivation for the present study is the fact that Leibnizadditive functions are generalizations of the arithmetic derivative $D$; namely, $D$ is Leibnizadditive with $h_{D}(n)=n$. We study the basic properties of Leibniz-additive functions and, among other things, show that a Leibniz-additive function $f$ is totally determined by the values of $f$ and $h_{f}$ at primes. We also find connections of Leibniz-additive functions to the usual product, composition and Dirichlet convolution of arithmetic functions. The arithmetic partial derivative is also considered.

Keywords: Arithmetic derivative, Arithmetic partial derivative, Arithmetic function, Completely additive function, Completely multiplicative function, Leibniz rule, Dirichlet convolution.

2010 Mathematics Subject Classification: 11A25, 11A41. 


\section{Introduction}

Let $n$ be a positive integer. Its arithmetic derivative $D(n)=n^{\prime}$ is defined as follows:

(i) $\quad p^{\prime}=1$ for all primes $p$,

(ii) $\quad(m n)^{\prime}=m n^{\prime}+m^{\prime} n$ for all positive integers $m$ and $n$.

Given

$$
n=\prod_{q \in \mathbb{P}} q^{\nu_{q}(n)},
$$

where $\mathbb{P}$ is the set of primes, the formula for computing the arithmetic derivative of $n$ is (see, e.g., $[2,13])$

$$
n^{\prime}=n \sum_{p \in \mathbb{P}} \frac{\nu_{p}(n)}{p} .
$$

A brief summary on the history of arithmetic derivative and its generalizations to other number sets can be found, e.g., in $[2,13,5]$.

Similarly, one can define the arithmetic partial derivative (see, e.g., [7, 5]) via

$$
D_{p}(n)=n_{p}^{\prime}=\frac{\nu_{p}(n)}{p} n
$$

and the arithmetic logarithmic derivative [13] as

$$
\operatorname{ld}(n)=\frac{D(n)}{n} .
$$

An arithmetic function $f$ is said to be additive if $f(m n)=f(m)+f(n)$, whenever $\operatorname{gcd}(m, n)=$ 1 , and multiplicative if $f(1)=1$ and $f(m n)=f(m) f(n)$, whenever $\operatorname{gcd}(m, n)=1$. Additive and multiplicative functions are totally determined by their values at prime powers. Further, an arithmetic function $f$ is said to be completely additive if $f(m n)=f(m)+f(n)$ for all positive integers $m$ and $n$, and completely multiplicative if $f(1)=1$ and $f(m n)=f(m) f(n)$ for all positive integers $m$ and $n$. These functions are widely studied in the literature, see, e.g., $[1,6,8,9,10,11,12]$.

We say that an arithmetic function $f$ is Leibniz-additive (or, L-additive, in short) if there is a completely multiplicative function $h_{f}$ such that

$$
f(m n)=f(m) h_{f}(n)+f(n) h_{f}(m)
$$

for all positive integers $m$ and $n$. Then $f(1)=0$, since $h_{f}(1)=1$. The property (1) may be considered a generalized Leibniz rule. This terminology arises from the observation that the arithmetic derivative $D$ is L-additive with $h_{D}(n)=n$; it satisfies the usual Leibniz rule

$$
D(m n)=D(m) n+D(n) m
$$

for all positive integers $m$ and $n$, and the function $h_{D}(n)=n$ is completely multiplicative. In addition, the arithmetic partial derivative with respect to the prime $p$ is L-additive with $h_{D_{p}}(n)=$ 
$n$. Further, all completely additive functions $f$ are L-additive with $h_{f}(n)=1$. For example, the logarithmic derivative of $n$ is completely additive, since

$$
\operatorname{ld}(m n)=\operatorname{ld}(m)+\operatorname{ld}(n)
$$

The term "L-additive function" seems to be new in the literature, yet Chawla [3] has defined the concept of a completely distributive arithmetic function meaning the same as we do with an L-additive function. However, this is a somewhat misleading term, since a distributive arithmetic function usually refers to the property that

$$
f(u * v)=(f u) *(f v),
$$

i.e., the function $f$ distributes over the Dirichlet convolution. This is satisfied by completely multiplicative arithmetic functions, not by completely distributive functions as Chawla defined them.

An arithmetic function $f$ is said to generalized additive [4] if there is a multiplicative function $h_{f}$ such that (1) holds for all positive integers $m$ and $n$ with $\operatorname{gcd}(m, n)=1$. Chawla [3] refers to these functions as distributive arithmetic functions. Some properties of these functions are presented in $[3,4]$. It is clear that L-additive functions is a subclass of the class of generalized additive functions, and, in this sense, we could refer to L-additive functions as generalized complete additive functions. We, however, prefer the term "L-additive function", since our main purpose is to attain new aspects on the arithmetic derivative and the arithmetic partial derivative. For the same reason, in this paper, we do not consider wider classes of arithmetic functions such as generalized additive functions, or multiplicative analogues of the present concepts.

In this paper, we consider L-additive functions especially from the viewpoint that they are generalizations of the arithmetic derivative and the arithmetic partial derivative. In Section 2, we study basic properties of L-additive functions and thereby provide some new insight into the arithmetic derivative and the arithmetic partial derivative. In Section 3, we study L-additivity, the arithmetic derivative and the arithmetic partial derivative in terms of the Dirichlet convolution.

\section{Basic properties}

Theorem 2.1. Let $f$ be an arithmetic function. If $f$ is $L$-additive and $h_{f}$ is nonzero-valued, then $f / h_{f}$ is completely additive. Conversely, if there is a completely multiplicative nonzero-valued function $h$ such that $f / h$ is completely additive, then $f$ is L-additive and $h_{f}=h$.

Proof. If $f$ satisfies (1) and $h_{f}$ is never zero, then

$$
\frac{f(m n)}{h_{f}(m n)}=\frac{f(m) h_{f}(n)+f(n) h_{f}(m)}{h_{f}(m) h_{f}(n)}=\frac{f(m)}{h_{f}(m)}+\frac{f(n)}{h_{f}(n)},
$$

verifying the first part. The second part follows by substituting $h_{f}=h$ in the above and exchanging the sides of the second equation. 
Theorem 2.2. Let $f$ be an arithmetic function. If $f$ is L-additive and $h_{f}$ is nonzero-valued, then

$$
f=g_{f} h_{f},
$$

where $g_{f}$ is completely additive. Conversely, if $f$ is of the form

$$
f=g h,
$$

where $g$ is completely additive and $h$ is completely multiplicative, then $f$ is L-additive with $h_{f}=$ $h$.

Proof. If $f$ is L-additive such that $h_{f}(n) \neq 0$ for all positive integers $n$, then denoting $g_{f}=f / h_{f}$ in Theorem 2.1 we obtain $f=g_{f} h_{f}$, where $g_{f}$ is completely additive.

Conversely, assume that $f$ is of the form (3). Then

$$
\begin{aligned}
f(m n) & =h(m) h(n)[g(m)+g(n)]=(h g)(m) h(n)+(h g)(n) h(m) \\
& =f(m) h(n)+f(n) h(m) .
\end{aligned}
$$

Example 2.1. If an arithmetic function $f$ satisfies the Leibniz rule

$$
f(m n)=f(m) n+f(n) m
$$

for all positive integers $m, n$, then $f(n)=g_{f}(n) n$, where $g_{f}$ is completely additive. Also the converse holds. In particular, the arithmetic derivative $D$ and the arithmetic partial derivative $D_{p}$ satisfy (4).

Theorem 2.2 shows that each L-additive function $f$ such that $h_{f}$ is always nonzero can be represented as a pair of a completely additive function $g_{f}$ and a completely multiplicative function $h_{f}$. In this case, we write $f=\left(g_{f}, h_{f}\right)$. However, if $h_{f}(p)=0$ for some prime $p$ and $f(p)$ is nonzero, then there is not any function $g_{f}$ such that $f=g_{f} h_{f}$ and, consequently, a representation of this kind is not possible.

On the other hand, the representation of an L-additive function as such a pair is not always unique either. For instance, if $f$ and $g_{f}$ are identically zero, then $h_{f}$ may be any completely multiplicative function. The next theorem will however show that this representation is unique whenever $f$ and $h_{f}$ are nonzero for all primes. Observe that the latter condition is equivalent to assuming that $h_{f}$ is nonzero for all positive integers.

Theorem 2.3. If $f$ is L-additive such that $f(p) \neq 0$ and $h_{f}(p) \neq 0$ for all primes $p$, then the representation $f=\left(g_{f}, h_{f}\right)$ is unique.

Proof. Let $f=\left(g_{f}, h_{f}\right)=\left(\tilde{g}_{f}, \tilde{h}_{f}\right)$. Then for all primes $p$,

$$
f(p)=g_{f}(p) h_{f}(p)=\tilde{g}_{f}(p) \tilde{h}_{f}(p) .
$$

On the other hand, by the definition of L-additivity,

$$
f\left(p^{2}\right)=2 f(p) h_{f}(p)=2 f(p) \tilde{h}_{f}(p),
$$

which implies that $h_{f}(p)=\tilde{h}_{f}(p)$ and, consequently, $g_{f}(p)=\tilde{g}_{f}(p)$. 
Example 2.2. The arithmetic derivative can be represented as $D=\left(g_{D}, h_{D}\right)$, where $g_{D}(p)=$ $1 / p=\operatorname{ld}(p)$ and $h_{D}(p)=p$ for all primes $p$. In other words, the arithmetic derivative has the representation as the pair of the logarithmic derivative and the identity function. Similarly, for the arithmetic partial derivative with respect to the prime $p$, we have $D_{p}=\left(g_{D_{p}}, h_{D_{p}}\right)$, where $g_{D_{p}}(q)=0$ if $q \neq p, g_{D_{p}}(p)=1 / p$, and $h_{D_{p}}(q)=q$ for all primes $q$ (that is, $h_{D_{p}}$ is the identity function).

Completely additive and completely multiplicative functions are totally determined by their values at primes as follows. Let

$$
n=q_{1} q_{2} \cdots q_{r}=p_{1}^{n_{1}} p_{2}^{n_{2}} \cdots p_{s}^{n_{s}}
$$

where $q_{1}, q_{2}, \ldots, q_{r}$ are primes and $p_{1}, p_{2}, \ldots, p_{s}$ are distinct primes. If $f$ is completely additive, then $f(1)=0$ and

$$
f(n)=\sum_{i=1}^{r} f\left(q_{i}\right)=\sum_{i=1}^{s} n_{i} f\left(p_{i}\right),
$$

and if $f$ is completely multiplicative, then $f(1)=1$ and

$$
f(n)=\prod_{i=1}^{r} f\left(q_{i}\right)=\prod_{i=1}^{s} f\left(p_{i}\right)^{n_{i}}
$$

If $f$ is only L-additive, then we must also know the values of $h_{f}$ at primes. A generalization of (6) (and an analogue of (7)) is given below.

Theorem 2.4. Let $n$ be as in (5). If $f$ is L-additive, then

$$
f(n)=\sum_{i=1}^{r} h_{f}\left(q_{1}\right) \cdots h_{f}\left(q_{i-1}\right) f\left(q_{i}\right) h_{f}\left(q_{i+1}\right) \cdots h_{f}\left(q_{r}\right) .
$$

If $h_{f}\left(p_{1}\right), \ldots, h_{f}\left(p_{s}\right) \neq 0$, then

$$
f(n)=h_{f}(n) \sum_{i=1}^{r} \frac{f\left(q_{i}\right)}{h_{f}\left(q_{i}\right)}=h_{f}(n) \sum_{i=1}^{s} \frac{n_{i} f\left(p_{i}\right)}{h_{f}\left(p_{i}\right)} .
$$

Proof. In order to verify the first claim, it suffices to notice that

$$
\begin{aligned}
f(n) & =f\left(q_{1}\right) h_{f}\left(q_{2} \cdots q_{r}\right)+f\left(q_{2} \cdots q_{r}\right) h_{f}\left(q_{1}\right) \\
& =f\left(q_{1}\right) h_{f}\left(q_{2}\right) \cdots h_{f}\left(q_{r}\right)+f\left(q_{2} \cdots q_{r}\right) h_{f}\left(q_{1}\right) \\
& =f\left(q_{1}\right) h_{f}\left(q_{2}\right) \cdots h_{f}\left(q_{r}\right)+h_{f}\left(q_{1}\right)\left[f\left(q_{2}\right) h_{f}\left(q_{3} \cdots q_{r}\right)+f\left(q_{3} \cdots q_{r}\right) h_{f}\left(q_{2}\right)\right] \\
& =f\left(q_{1}\right) h_{f}\left(q_{2}\right) \cdots h_{f}\left(q_{r}\right)+h_{f}\left(q_{1}\right)\left[f\left(q_{2}\right) h_{f}\left(q_{3}\right) \cdots h_{f}\left(q_{r}\right)+f\left(q_{3} \cdots q_{r}\right) h_{f}\left(q_{2}\right)\right] \\
& =\cdots \\
& =\sum_{i=1}^{r} h_{f}\left(q_{1}\right) \cdots h_{f}\left(q_{i-1}\right) f\left(q_{i}\right) h_{f}\left(q_{i+1}\right) \cdots h_{f}\left(q_{r}\right) .
\end{aligned}
$$

The rest of the theorem follows from the equation $h_{f}(n)=h_{f}\left(q_{1}\right) \cdots h_{f}\left(q_{r}\right)$, which holds, since $h_{f}$ is completely multiplicative. 
Example 2.3. Since $D(p)=1$ and $h_{D}(p)=p$ for all primes $p$, Theorem 2.4 gives the well-known formula

$$
D(n)=\sum_{i=1}^{r} q_{1} \cdots q_{i-1} q_{i+1} \cdots q_{r}=n \sum_{i=1}^{r} \frac{1}{q_{i}}=n \sum_{i=1}^{s} \frac{n_{i}}{p_{i}} .
$$

It also implies that

$$
f\left(p^{k}\right)=k h_{f}(p)^{k-1} f(p)
$$

for all primes $p$ and nonnegative integers $k$. (For $k=0, h_{f}(p)$ must be nonzero.) In particular, for the arithmetic derivative, this reads

$$
D\left(p^{k}\right)=k p^{k-1}
$$

For the arithmetic partial derivative $D_{p}$, Theorem 2.4 reduces to its definition.

Theorem 2.5. If $u$ is L-additive and $v$ is completely multiplicative, then their product function uv is L-additive with $h_{u v}=h_{u} v$.

Proof. For all positive integers $m$ and $n$,

$$
\begin{aligned}
(u v)(m n) & =u(m n) v(m n)=\left(u(m) h_{u}(n)+u(n) h_{u}(m)\right) v(m) v(n) \\
& =(u v)(m)\left(h_{u} v\right)(n)+(u v)(n)\left(h_{u} v\right)(m) .
\end{aligned}
$$

Thus $u v$ is L-additive with completely multiplicative part equaling to $h_{u} v$.

Example 2.4. The function $f(n)=D(n) n^{k}$, where $k$ is a nonnegative integer, is L-additive with $h_{f}(n)=n^{k+1}$. The same property holds for the arithmetic partial derivative $D_{p}$, since $h_{D}=h_{D_{p}}$.

Theorem 2.6. If $v$ is L-additive and $u$ is completely multiplicative with positive integer values, then their composite function $v \circ u$ is L-additive with $h_{v \circ u}=h_{v} \circ u$.

Proof. For all positive integers $m$ and $n$,

$$
\begin{aligned}
(v \circ u)(m n) & =v(u(m n))=v(u(m) u(n))=v(u(m)) h_{v}(u(n))+v(u(n)) h_{v}(u(m)) \\
& =(v \circ u)(m)\left(h_{v} \circ u\right)(n)+(v \circ u)(n)\left(h_{v} \circ u\right)(m) .
\end{aligned}
$$

It remains to show that $h_{v} \circ u$ is completely multiplicative. We have

$$
\left(h_{v} \circ u\right)(m n)=h_{v}(u(m n))=h_{v}(u(m) u(n))=h_{v}(u(m)) h_{v}(u(n))=\left(h_{v} \circ u\right)(m)\left(h_{v} \circ u\right)(n) .
$$

This completes the proof.

Example 2.5. Let $u$ be as in Theorem 2.6. Since $D$ is L-additive, then, by this theorem, $D \circ u$ is L-additive with $h_{D \circ u}=u$; that is,

$$
D(u(m n))=u(n) D(u(m))+u(m) D(u(n))
$$

for all positive integers $m, n$. In particular,

$$
D\left((m n)^{k}\right)=n^{k} D\left(m^{k}\right)+m^{k} D\left(n^{k}\right),
$$

where $k$ is a nonnegative integer. These equations follow also from the fact that $D$ satisfies the Leibniz rule. The above formulas also hold for the arithmetic partial derivative $D_{p}$. 


\section{L-additive functions in terms of the Dirichlet convolution}

Above we have seen that many fundamental properties of the arithmetic derivative, e.g., the formula for computing the arithmetic derivative of a given positive integer, are rooted to the fact that $D$ is L-additive. We complete this article by changing our point of view slightly and demonstrate that L-additive functions can also be studied in terms of the Dirichlet convolutions.

Let $u$ and $v$ be arithmetic functions. Their Dirichlet convolution is

$$
(u * v)(n)=\sum_{d \mid n} u(d) v(n / d)
$$

We let $f(u * v)$ denote the product function of $f$ and $u * v$, i.e.,

$$
(f(u * v))(n)=f(n)(u * v)(n) .
$$

Theorem 3.1. An arithmetic function $f$ is completely additive if and only if

$$
f(u * v)=(f u) * v+u *(f v)
$$

for all arithmetic functions $u$ and $v$.

Proof. See [11, Proposition 2].

The next theorems show that L-additive functions can also be characterized in an analogous way.

Theorem 3.2. Let $f$ be an arithmetic function. If $f$ is L-additive and $h_{f}$ is nonzero-valued, then

$$
f(u * v)=(f u) *\left(h_{f} v\right)+\left(h_{f} u\right) *(f v)
$$

for all arithmetic functions $u$ and $v$. Conversely, if there is a completely multiplicative nonzerovalued function $h$ such that

$$
f(u * v)=(f u) *(h v)+(h u) *(f v)
$$

for all arithmetic functions $u$ and $v$, then $f$ is L-additive and $h_{f}=h$.

Proof. Under the assumptions of the first part, Theorems 2.1 and 3.1 imply

$$
\left(f / h_{f}\right)(u * v)=\left(f u / h_{f}\right) * v+u *\left(f v / h_{f}\right) .
$$

Multiplying by $h_{f}$, the left-hand side becomes $f(u * v)$. Since

$$
h_{f}\left(\left(f u / h_{f}\right) * v\right)=(f u) *\left(h_{f} v\right), \quad h_{f}\left(u *\left(f v / h_{f}\right)\right)=\left(h_{f} u\right) *(f v)
$$

by (2), the claim follows. To prove the second part, multiply (9) by $1 / h$ and perform a simple modification of the above. 
Corollary 3.1. If $u$ and $v$ are arithmetic functions, then

$$
D(u * v)=(D u) *(N v)+(N u) *(D v)
$$

where $N(n)=n$.

Proof. It suffices to notice that $h_{D}=N$.

On the other hand, taking $u=v$, equation (8) becomes

$$
f(u * u)=2\left[(f u) *\left(h_{f} u\right)\right] .
$$

For $u=E$, where $E(n)=1$ for all positive integers $n$, this reads

$$
f \tau=2\left(f * h_{f}\right),
$$

where $\tau$ is the divisor-number-function. Especially, we have

$$
D(u * u)=2[(D u) *(N u)]
$$

and, with $u=E$,

$$
D \tau=2(D * N)
$$

Some remarks can be made also in the opposite direction. Assume now that

$$
f \tau=2(f * h)
$$

for some completely multiplicative function $h$ that is nonzero for all positive integers. Then

$$
(f / h) \tau=2((f / h) * E) .
$$

Thus, again according to [11, Proposition 2], we see that $f / h$ is completely additive, which shows that $f$ is L-additive with $h_{f}=h$. In particular, if

$$
f \tau=2(f * N)
$$

then $f$ is L-additive with $h_{f}=N$. For example, $D$ satisfies this condition.

All of the above results for the arithmetic derivative $D$ hold also for the arithmetic partial derivative $D_{p}$, since $h_{D}=h_{D_{p}}$.

Further properties of L-additive functions in terms of the Dirichlet convolution can be derived from the results in $[8,11]$. It would be possible to obtain some properties of L-additive functions in terms of the unitary convolution as well from the results in [8].

\section{References}

[1] Apostol, T. M. (1976) Introduction to Analytic Number Theory, Springer-Verlag, New York.

[2] Barbeau, E. J. (1961) Remarks on an arithmetic derivative, Canad. Math. Bull. 4(2), 117-122. 
[3] Chawla, L. M. (1973) A note on distributive arithmetical functions, J. Nat. Sci. Math. 13(1), $11-17$.

[4] Haukkanen, P. (1992) A note on generalized multiplicative and generalized additive arithmetic functions, Math. Stud. 61, 113-116.

[5] Haukkanen, P., Merikoski, J. K. \& Tossavainen, T. (2016) On arithmetic partial differential equations, J. Integer Seq. 19, Article 16.8.6.

[6] Kiuchi, I. \& Minamide, M. (2014) On the Dirichlet convolution of completely additive functions. J. Integer Seq. 17, Article 14.8.7.

[7] Kovič, J. (2012) The arithmetic derivative and antiderivative, J. Integer Seq. 15, Article 12.3.8.

[8] Laohakosol, V. \& Tangsupphathawat, P. (2016) Characterizations of additive functions. Lith. Math. J. 56(4), 518-528.

[9] McCarthy, P. J. (1986) Introduction to Arithmetical Functions, Springer-Verlag, New York.

[10] Sándor, J. \& Crstici, B. (2004) Handbook of Number Theory II, Kluwer Academic, Dordrecht.

[11] Schwab, E. D. (1995) Dirichlet product and completely additive arithmetical functions, Nieuw Arch. Wisk. 13(2), 187-193.

[12] Shapiro, H. N. (1983) Introduction to the Theory of Numbers, Wiley InterScience, New York.

[13] Ufnarovski, V. \& Åhlander, B. (2003) How to differentiate a number, J. Integer Seq. 6, Article 03.3.4. 